\title{
Dengue Typhoid Co-infection: a New Threat
}

\author{
*MMR Siddiqui ${ }^{1}$, QT Islam² ${ }^{2}$ MS Islam ${ }^{3}$
}

\begin{abstract}
Acute febrile illness is the most common clinical presentation among patients attending to doctor in developing countries. In Bangladesh, dengue and typhoid fever have emerged as major public health problems. Co-infection with both these diseases is rarely reported and are known to present with overlapping symptoms making the clinical diagnosis difficult. The exact incidence of dengue and typhoid co-infection is not known. Here we report a case of co-infection of dengue fever with typhoid fever.
\end{abstract}

Key Words: Dengue, fever, typhoid, co-infection.

\section{Introduction}

Emerging and re-emerging diseases are a major concern in Asia due to population growth, urbanization, and global migration. Acute febrile illness is the most common clinical presentation among patients attending to doctor in developing countries. ${ }^{1,2}$ In our Bangladesh most common cause of acute febrile illness in urban area are Dengue, Typhoid fever and Viral Hepatitis. In Bangladesh, dengue and typhoid fever have emerged as major public health problems. Co-infections of dengue with Chikungunya, malaria and other arboviruses have been frequently reported from endemic areas. ${ }^{3}$ But Co-infection of Dengue and Typhoid fever is rarely reported and are known to present with overlapping symptoms making the clinical diagnosis difficult. $1,2,3$ The exact incidence of dengue and typhoid coinfection is not known. Dual infections tend to have prolonged fever, increased risk of complications and delayed in diagnosis. 4 Here we report a case of coinfection of dengue with typhoid fever.

\section{Case Report}

A 21-year-old boy presented with high grade fever for 4 days, associated with severe headache, myalgia, nausea and prostration. Fever was continued in nature and does not subsided with paracetamol. On examination he had generalized itchy maculo-papular skin rashes over face, trunk and limbs. He was febrile with body temperature $102^{0} \mathrm{~F}$. Significant findings were a positive tourniquet test. Other system examinations were unremarkable. Investigations revealed, there was a significant decrease in platelet count $\left(80 \times 10^{9} / \mu \mathrm{l}\right)$. Total White Blood Cell (WBC) count 3240/ $\mu$ l and NS1 antigen for dengue was positive. But no features of plasma leakage. With all of these findings he was diagnosed as a case of Dengue Fever. He was treated conservatively with plenty of oral fluids, bed rest, paracetamol and antihistamine.

But from the 8th day he developed loose motion and pain abdomen. However, in spite of apparent clinical improvement (decrease myalgia and skin rash), the fever spikes persisted. In view of persisting fever, blood samples were sent for culture considering the possibility of scrub typhus and typhoid fever. We repeat Complete Blood Count (CBC) revealed platelet count $130 \times 10^{9} / \mu \mathrm{l}$ and $\mathrm{WBC}$ was $5410 / \mu \mathrm{l}$. After 3 days blood culture and sensitivity report came and revealed growth of Salmonella typhi with sensitivity to Cefixim, Ceftriaxone, Cefepime, Azithromycin and Meropenem. As we found as a case of Dengue and Typhoid co-infection, we have

${ }_{1} *$ Dr. Md. Mahmudur Rahman Siddiqui, Associate Professor of Medicine, Anwer Khan Modern Medical College \& Hospital, Dhaka.e-mail: dr.mahmud99@yahoo.com

2Prof. Quazi Tarikul Islam, Professor of Medicine, Popular Medical College \& Hospital, Dhaka ${ }^{3}$ Dr. Mohammad Shahidul Islam, Registrar, Department of Medicine, Anwer Khan Modern Medical College \& Hospital, Dhaka

*Corresponding Author

Date of submission: 20.09.2018 Date of acceptance: 15.11.2018

AKMMC J 2019; 10(1) : 96-97 
started oral Cefixim, $400 \mathrm{mg}$ b.i.d. for 14 days and he showed dramatic improvement.

\section{Discussion}

Infectious diseases are one of the major causes of morbidity and mortality in developing countries. ${ }^{3}$ Concurrent infections with multiple infectious agents make the correct diagnosis and management a challenging task. In Bangladesh, dengue virus causes epidemic and sporadic cases year-round, with a peak from May to November, during the monsoon season. 2 Typhoid fever usually presents with progressive prolonged high fever without returning to normal. ${ }^{2}$ The fever rises in increments like step leader and usually reaches $40-40.5^{\circ} \mathrm{C}$ by the end of the first week of illness. 5 Particularly in dengue, there is a sudden sharp rise in temperature between $39^{\circ} \mathrm{C}$ and $40^{\circ} \mathrm{C}$, and this is frequently accompanied by a flushed face, severe headache, myalgia, arthralgia, and rashes. 6 The fever may be biphasic, lasting 5-7 days in the majority of cases. In typhoid fever, a dull, continuous frontal headache beginning during the first few days of fever along with gastrointestinal symptoms, such as diarrhea and constipation. ${ }^{7}$

It is a known fact that bacterial infections follow viral diseases especially in upper respiratory diseases, the effect of one disease over the other is not exactly known in dengue-typhoid co-infections. ${ }^{8}$ The reasons for bacterial co-infections in some patients with dengue are also not yet fully known. It is known that dengue virus can cause a diminished $\mathrm{T}$ cell proliferation in response to mitogens in vitro. ${ }^{2} \mathrm{~A}$ possible interaction between dengue and typhoid may arise through intestinal endothelial damage or intestinal haemorrhage, or through immunosuppression superimposed by initial virus illness. 9 Another probable reason cited for increased gram negative sepsis with dengue is the breakdown of intestinal mucosal barrier. ${ }^{2}$

However, fever persisted in our patient despite signs of recovery from dengue, such as improvement in headache, myalgia, skin rash and platelet count. According to the WHO, a Dengue fever usually lasts for 2-7 days. 10 However, our patient continued to have fever for $>7$ days, made us suspicious for differential diagnosis. Both the diseases are endemic in Bangladesh, they remain public health problems, and it is possible to have both infections at the same time. Both dengue and typhoid may lead to many complications if not diagnosed and treated promptly.
There is a need to document such cases and heighten awareness among clinicians to the changing patterns of infectious disease manifestations.

\section{Conclusion}

Co-infection should always be considered in endemic areas while dealing with patients with prolonged acute nonresponsive febrile illness. In every febrile patients common infections should be evaluated with through history, clinical examinations and logical investigations. If not treated early, dengue and typhoid together, may lead to multi-organ involvement with significant morbidity and mortality.

Conflict of interest: We have no conflict of interest.

\section{References}

1. Srinivasaraghavan R, Narayanan P, Kanimozhi T. Typhoid coinfection in a child with dengue. J Infect Dev Ctries 2015; 9(9): 1033-1035. doi:10. 3855/jidc. 5230.

2. Jagadishkumar K, Shareef M, Hosur D et al. Dengue and Typhoid Fever Coinfection in A Child. J Pediatr Inf 2016; 10: $36-8$.

3. Vigna SRV, Gopalsamy S, Padma S. Dengue and typhoid coinfection: A case report from a tertiary care hospital in South India. Int J Case Rep Images 2016; 7(9): 563-565.

4. Bansal R, Bansal P, Tomar LR. Typhoid and dengue coinfection: case reports. Trop Doct 2015 Jan; 45(1): 52-3.

5. Basuki PS. Concurrent dengue infection and enteric fever. A case series. Folia Medica Indonesiana 2003; 39: 54-60.

6. Comprehensive Guidelines for Prevention and Control of Dengue and Dengue Haemorrhagic Fever Revised and expanded edition ${ }^{\circledR}$ World Health Organization 2011. Printed in India. Available from: URL: http://www.searo.who. int/entity/vector_borne_tropical_diseases/ documents /SEAROTPS60/en/.

7. Sharma Y, Arya V, Jain S, et al. Dengue and Typhoid Coinfection-Study from a Government Hospital in North Delhi. J Clin Diagn Res 2014; 8: 9-11. [Cross Ref]

8. Sharma Y, Arya V, Jain S, et al. Dengue and Typhoid Coinfection- Study from a Government Hospital in North Delhi. J Clin Diagn Res 2014 Dec; 8(12): DC09-11.

9. Lee IK, Liu JW and Yang KD. Clinical characteristics and risk factors for concurrent bacteremia in adults with dengue hemorrhage fever. Am J Trop Med Hyg 2005; 72: 221-226.

10. Rigau-Perez JG, Clark GC, Gubler DJ, et al. Dengue and dengue haemorrhagic fever. Lancet 1998; 352: 971-977. 